\title{
Outcome of relapsed infant acute lymphoblastic leukemia treated on the interfant-99 protocol
}

EMC Driessen, P de Lorenzo, M Campbell, M Felice, A Ferster, I Hann, A Vora, L Hovi, G Escherich, CK Li, G Mann, T Leblanc, F Locatelli, A Biondi, J Rubnitz, M Schrappe, L Silverman, J Stary, R Suppiah, T Szczepanski, M Valsecchi and R Pieters

Correction to: Leukemia (2016) 30, 1184-1187; doi:10.1038/ leu.2015.246

Following the publication of this article the authors noted that the labels in Figures $1 \mathrm{~b}$ and $\mathrm{d}$ have been switched. The correct labels in Figure 1b are; mixed lineage leukemia rearranged
(MLL rearranged; dotted line) and unknown (thin solid line). In Figure 1d the correct labels are; bone marrow involvement (BM involv; dotted line) and isolated extramedullary (Isolated Ext; thin solid line).

The authors wish to apologize for any inconvenience caused.

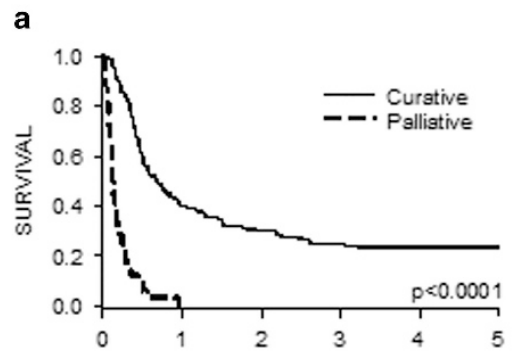

b
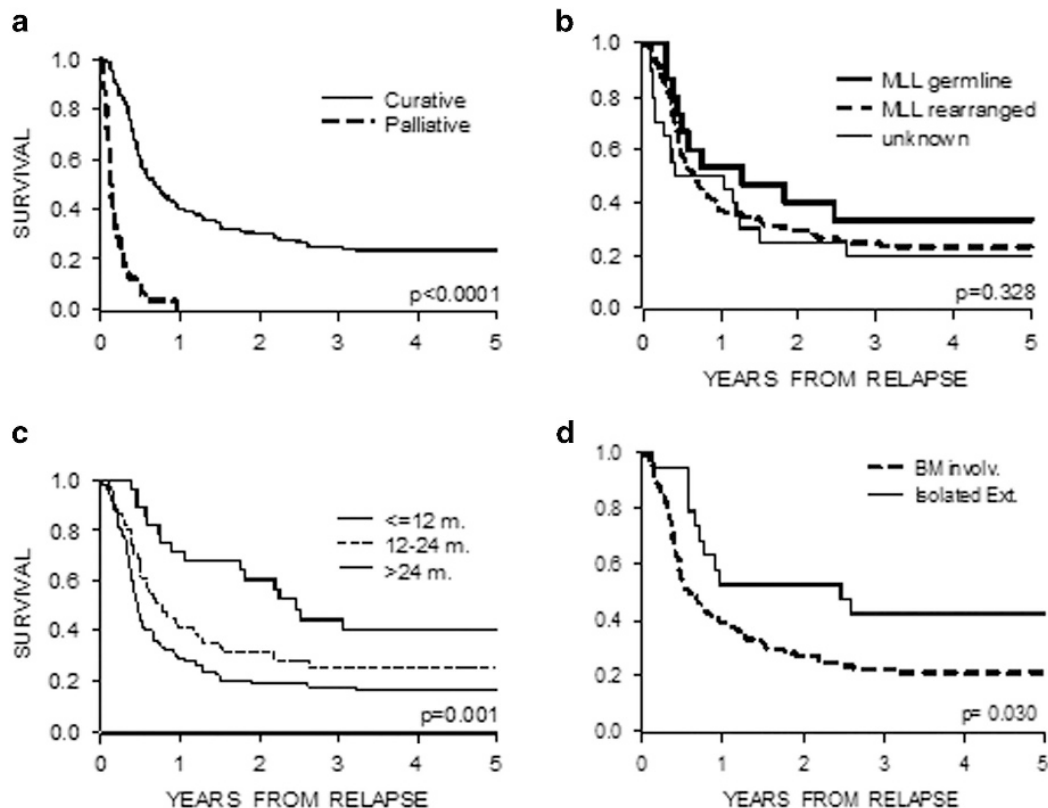

d

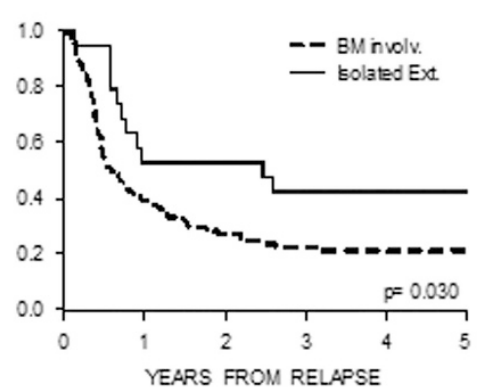

e

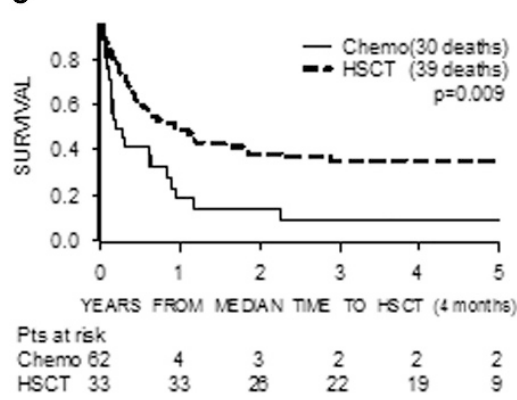

f

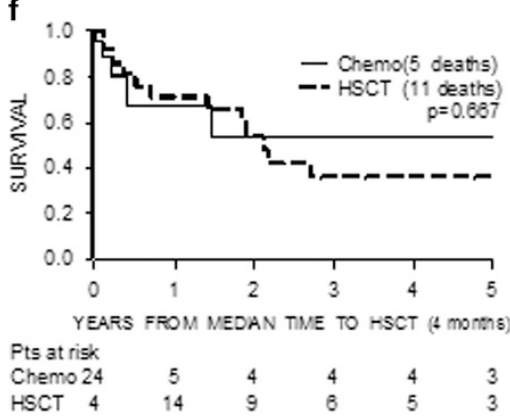

Figure 1. Overall survival, defined as time from relapse until death, (a) by treatment given after relapse, (b) of patients treated with curative intents by MLL (mixed lineage leukemia) status at initial diagnosis, (c) of patients treated with curative intents by time at relapse after initial diagnosis, (d) of patients treated with curative intents by site at relapse, (e) of patients treated with HSCT (hematopoietic stem cell transplantation) vs chemotherapy only of early relapses (within 24 months from initial diagnosis), (f) of patients treated with HSCT vs chemotherapy only of late relapses (beyond 24 months from initial diagnosis). Curves were estimated using the Kaplan-Meier method and analyzed by Log-rank tests. Follow-up was updated at 31 December 2009 and median follow-up (range) was 5.2 years (1 month-10.1 years). Survival analyses regarding HSCT were corrected for waiting time to transplantation. BM involv, bone marrow involvement; chemo, chemotherapy; HSCT, hematopoietic stem cell transplantation; Isolated Ext, isolated extramedullary; m, months; MLL, mixed lineage leukemia; MLL germline, cases without MLL translocation; MLL rearranged, cases with MLL translocation; unknown, cases with unknown MLL status. 